\title{
Abastecimiento de medicamentos en unidades de primer nivel de atención de la Secretaría de Salud de México
}

\author{
0 ctavio Gómez-D antés, M.C., M.S.P., ${ }^{(1)}$ Francisco Garrido-Latorre, M.C., M. en C., ${ }^{(1)}$ \\ Laura LeticiaT irado-Gómez, M.C., M. en C., ${ }^{(2)}$ D olores Ramírez, M.C ., M., M. en C.,1(2) \\ Claudia Macías, M.C., M. en C. ${ }^{(2)}$
}

\section{Gómez-Dantés O, Garrido-Latorre F, Tirado-Gómez LL, Ramírez D, Macías C. Abastecimiento de medicamentos en unidades de primer nivel de atención de la Secretaría de Salud de México. Salud Publica Mex 2001;43:224-232.} El texto completo en inglés de este artículo está disponible en: http://www.insp.mx/salud/index.html

\section{Resumen}

Objetivo. Conocer el nivel de disponibilidad de algunos medicamentos esenciales (ME) en las unidades de primer nivel de atención de la Secretaría de Salud de México (SSA), a partir de una investigación realizada en 1996-1997. Material y métodos. Del universo de jurisdicciones sanitarias de los 18 estados participantes en el Programa de Ampliación de Cobertura (PAC) se construyó una muestra de manera aleatoria. Todas las unidades de primer nivel de atención ubicadas en las jurisdicciones seleccionadas fueron evaluadas mediante una inspección que utilizó una guía que contenía una lista de $36 \mathrm{ME}$. En una primera fase del análisis se calcularon el número absoluto y la proporción de unidades que no contaban con un solo ejemplar de alguno de los ME de la lista. En las unidades con disponibilidad de medicamentos se calculó la mediana de la distribución. En una segunda fase se realizó la sumatoria por separado de los 36 medicamentos y de los 10 insumos, y se obtuvieron las medias diferenciadas por tipo de centro de salud y por estado. El estadístico utilizado para la comparación de las medias fue la prueba de Scheffé mediante el análisis de varianza de una vía. Posteriormente se agruparon los medicamentos de acuerdo con sus principales tipos de indicación, y se calcularon las medias y las proporciones de medicamentos disponibles. Las diferencias de proporciones se evaluaron con el estadístico ji cuadrada. Resultados En el momento de la inspección, las unidades visitadas conta-
Gómez-Dantés O, Garrido-Latorre F,

Tirado-Gómez LL, Ramírez D, Macías C.

Drug supplies at Ministry

of Health primary health

care units in Mexico.

Salud Publica Mex 2001;43:224-232.

The English version of this paper

is available at: http://www.insp.mx/salud/index.html

\begin{abstract}
A bstract
Objective.To identify the availability of some essential drugs (ED) at primary health care units of the Mexican Ministry of Health (SSA), using data from a research work conducted in 1996 and 1997. Materials and methods $A$ random sample of the 18 sanitary jurisdictions of states participating in the Extension of C overage Program (ECP) was drawn. All primary care units from selected jurisdictions were evaluated through an inspection visit, using a checklist that included $36 \mathrm{ED}$. In phase I of the analysis, the absolute number and proportion of units without a single item of any of the 36 drugs were calculated. In the units with available drugs, the median of the distribution was also calculated. In phase II, the medians according to the type of health unit and state was obtained. The statistic utilized for the comparison of the medians was the Scheffé test with one way variance anlysis. In addition, the drugs were classified according to their therapeutic indication and the medians and proportion of available drugs were calcultaed. The differences in proportion were evaluated with the statistic $\chi^{2}$. Results. During the visits, on average, 18 of the 36 drugs included in the list of the study were found in the health units. The availability of antibiotics, antituberculosis drugs, and antimalarial drugs was particularly poor. In contrast, oral rehydration salts, family planning methods and vaccines were usually available. In general, the PAC 3 states presented the best availability figures. Conclusions. The
\end{abstract}

(1) Dirección General de Evaluación del Desempeño, Secretaría de Salud, México.

(2) Centro de Investigación en Sistemas de Salud, Instituto N acional de Salud Pública, México.

Fecha de recibido: 1 de febrero de 2001 - Fecha de aprobado: 4 de abril de 2001 Solicitud de sobretiros: 0 ctavio Gómez-D antés. Leibnitz N 0. 20,50. piso, colonia Anzures, 11590 México, D.F., México. Correo electrónico:ogómez@ mail.ssa.gob.mex 
ban en promedio, con sólo 18 de los 36 medicamentos incluidos en el estudio. Los problemas de abastecimiento de antibióticos, antifímicos y antipalúdicos fueron particularmente graves. En contraste, el abasto de sales de rehidratación oral, métodos de planificación familiar y biológicos fue relativamente aceptable. En general, los estados clasificados como PAC 3 presentaron las mejores cifras de abastecimiento. Conclusiones La SSA de México, en general, y el Programa de A mpliación de Cobertura, en particular, deberán redoblar esfuerzos para acabar con los cuellos de botella que impiden un abastecimiento adecuado de medicamentos esenciales en las unidades de primer nivel de atención. De otra manera, todas las demás actividades dirigidas a atender las necesidades de salud de las poblaciones más mar ginadas del país resultarán inútiles, ya que el acceso a los medicamentos constituye la pieza final indispensable de la enorme cadena de la atención a la salud. El texto completo en inglés de este artículo está disponible en: http://www.insp.mx/salud/index.html

Palabras clave: medicamentos esenciales; abastecimiento; México
Ministry of Health of México will have to develop an enormous effort to overcome the obstacles related to the supply of essential drugs in primary health care units. 0 therwise, all other efforts directed to meet the needs of the noninsured population will end up being useless, and enormous amounts of the already scarce resources of the health sector will be wasted since drugs are a vital component of the long chain of health care.The English version of this paper is available at: http://www.insp.mx/salud/index.html

Key words: essential drugs; availability; Mexico os medicamentos esenciales se definen como aquéllos de importancia vital que deben estar disponibles, en todo momento, en las dosis adecuadas y en cantidades suficientes para satisfacer las necesidades fundamentales de salud de todos los segmentos de la población. ${ }^{1}$

El concepto de medicamento esencial y las listas nacionales e internacionales de medicamentos esenciales surgieron en respuesta a cuatro grandes problemas endémicos: a) la carencia de medicamentos en las áreas rurales y urbanas marginadas; b) la proliferación de medicamentos de dudosa efectividad; c) los gastos crecientes en medicamentos, y d) los serios problemas de prescripción detectados en todas partes del mundo.

En México, la historia del uso de los medicamentos esenciales data de 1950, fecha en la que el Instituto Mexicano del Seguro Social (IMSS) crea su primera lista de medicamentos básicos. ${ }^{2}$ Veinticinco años después, en 1975, se publicó el Acuerdo Presidencial que establece que todas las instituciones públicas de salud deben contar con un Cuadro Básico de Medicamentos (CBM) de uso obligatorio. Sin embargo, no es sino hasta 1977 cuando se publica el primer cuadro básico de carácter sectorial. ${ }^{3}$ Sus objetivos fueron los siguientes: a) racionalizar el uso de medicamentos; b) impulsar el desarrollo de la industria farmacéutica nacional; c) mejorar el abasto de materias primas farmacológicas, y d) garantizar el acceso de todo el sector público de la salud a los medicamentos esenciales. ${ }^{4}$

Posteriormente, en 1983, también por Acuerdo Presidencial y con el fin explícito de racionalizar la ad- quisición, utilización y distribución de los insumos que se requieren para la prestación de los servicios de salud, se instituyó el Cuadro Básico de Insumos del Sector Salud (CBI). Este cuadro incluye, además del CBM, cuadros básicos de productos biológicos y reactivos de laboratorio, instrumental, equipo médico, material de curación y prótesis. $^{5}$ Además, se creó la Comisión Interinstitucional del CBI del Sector Salud, encargada de revisar periódicamente los listados de medicamentos y otros productos del CBI.

Estas medidas, sin embargo, no han garantizado un acceso razonable de la población no asegurada de México a los productos del CBI. De hecho, la escasa información disponible habla de serios problemas de abastecimiento. En la Encuesta Nacional de Salud II (ENSA II), ${ }^{*}$ los usuarios de los servicios de salud de la Secretaría de Salud de México (SSA) ${ }^{\ddagger}$ de tres de las cinco regiones en las que se dividió al país para la aplicación de la encuesta, refieren como principal motivo por el cual no regresarían a hacer uso de dichos servi-

* En 1994, como parte del esfuerzo por consolidar un Sistema Nacional de Encuestas de Salud, la Secretaría de Salud de México llevó a cabo una segunda encuesta nacional de salud con el fin de dar respuesta a las demandas de información sobre morbilidad, utilización y financiamiento de servicios, niveles de cobertura y calidad de la atención en México.

* La Secretaría de Salud de México tiene la responsabilidad de atender las necesidades de salud de la población no asegurada del país, que comprende entre 50 y $60 \%$ de la población nacional total. 
cios la falta o mala calidad de los medicamentos. ${ }^{6} \mathrm{La}$ misma ENSA II refiere que más del 50\% de los usuarios de los servicios de salud de la SSA, en el ámbito nacional $(52.5 \%)$, obtienen sus medicamentos en las farmacias privadas y no en el sitio de atención.

Fueron estos datos y los recuentos anecdóticos sobre los problemas de acceso a los medicamentos esenciales en México -que han aparecido en la prensa nacional y que los operadores de los servicios conocen en detalle- los que impulsaron la realización de una encuesta sobre el abastecimiento de medicamentos y material de curación en las unidades de primer nivel de atención del país. Esta encuesta se llevó a cabo en 18 estados de la República mexicana; su objetivo fue conocer el nivel de disponibilidad de algunos de los productos clave del CBI, en las unidades de primer nivel de atención de la SSA de los estados que participaron en un programa de ampliación de cobertura. Esta encuesta también podría servir de referencia para evaluar el impacto de los esfuerzos posteriores por solucionar los problemas de abastecimiento de medicamentos y otros insumos en estas unidades asistenciales de la SSA.

\section{Material y métodos}

El estudio cuyos resultados aquí se presentan fue de corte transversal, realizado en marzo-abril de 1996 y enero-marzo de 1997, en 466 unidades del primer nivel de atención de las 18 entidades federativas que participaron en el Programa de Ampliación de Cobertura (PAC) de la SSA. Dicho programa está dirigido a ofrecer 13 intervenciones de salud (saneamiento básico en el ámbito familiar; planificación familiar; atención prenatal, del parto y puerperio, y del recién nacido; vigilancia de la nutrición y crecimiento infantil; inmunizaciones; manejo de diarrea en el hogar; tratamiento antiparasitario de las familias; manejo de infecciones respiratorias agudas; prevención y control de tuberculosis pulmonar; prevención y control de la hipertensión arterial y de la diabetes mellitus; prevención de accidentes y manejo inicial de lesiones, y capacitación comunitaria para el autocuidado de la salud) a los 10 millones de mexicanos que a principios de 1995 no contaban con acceso regular a servicios básicos de salud.

A los estados participantes en este estudio se les ubicó en tres regiones: estados que participaron en un programa previo de ampliación de cobertura (Chiapas, Guerrero, Hidalgo y Oaxaca, región $\mathrm{PAC}_{1}$ ); estados que se incorporaron desde el primer año al PAC (Campeche, Michoacán, Nayarit, Puebla, Veracruz, Yucatán y Zacatecas, región $\mathrm{PAC}_{2}$ ), y estados que se incorpo- raron al PAC en el año dos del programa (Chihuahua, Durango, Guanajuato, Estado de México, Querétaro, San Luis Potosí y Sinaloa, región $\mathrm{PAC}_{3}$ ).

Ante la imposibilidad de estudiar la totalidad de las unidades ubicadas en las áreas beneficiarias del programa, se decidió constituir una muestra de jurisdicciones sanitarias seleccionadas de manera aleatoria. $\mathrm{La}$ jurisdicción sanitaria es la unidad básica de la SSA, y su función es la administración de los recursos y la operación de los servicios en un territorio específico. Para cada una de las 18 entidades federativas participantes en el PAC se eligió una jurisdicción y se estudiaron todas las unidades médicas de primer nivel de atención ubicadas en dicha unidad administrativa. Del total de unidades estudiadas, 242 (51.9\%) fueron centros de salud rurales dispersos (CSRD); 83 $(17.7 \%)$, centros de salud rurales concentrados (CSRC); $40(8.5 \%)$, centros de salud urbanos (CSU), y 101 $(21.6 \%)$, unidades auxiliares de salud (UAS).

Por medio de una inspección de las unidades y una entrevista con los proveedores de servicios se estudiaron las condiciones físicas de la unidad, la disponibilidad de medicamentos e insumos, y la existencia de programas preventivos y curativos. En este trabajo se presentan únicamente los resultados del estudio de disponibilidad de medicamentos e insumos.

Para la inspección se utilizó una guía de observación que contenía una lista de 36 medicamentos incluidos en el CBI, y que a juicio de los investigadores responsables del diagnóstico basal del PAC, resultaban imprescindibles para el control de enfermedades diarreicas y respiratorias agudas, enfermedades prevenibles por vacunación, tuberculosis pulmonar, diabetes mellitus, hipertensión arterial y paludismo. Asimismo, se incluyeron en la lista 10 insumos necesarios para la aplicación de inyecciones y para curaciones diversas (cuadro I).

En una primera fase del análisis estadístico se calcularon el número absoluto y la proporción de unidades que no contaban con un solo ejemplar de alguno de los medicamentos e insumos incluidos en la lista al momento de la inspección. En las unidades con disponibilidad de medicamentos se calculó la mediana de la distribución con su respectivo intervalo de confianza al 95\%. En una segunda fase se realizó la sumatoria por separado de los 36 medicamentos y de los 10 insumos, y se obtuvieron las medias diferenciadas por tipo de centro de salud y por estados. El estadístico utilizado para la comparación de las medias fue la prueba de Scheffé, mediante el análisis de varianza de una vía. Posteriormente se agruparon los medicamentos de acuerdo con sus principales tipos de indicación, a saber: antibióticos y sulfas, antiparasi- 


\section{Cuadro I \\ Medicamentos esenciales disponibles POR tIPO DE UNIDAD DE PRIMER NIVEL DE ATENCIÓN. Secretaría de Salud, México, 1996-1997}

$\begin{array}{lccccc}\text { Medicamentos/unidades } & \operatorname{CSU}(n=40) & \operatorname{CSRC}(n=83) & \operatorname{CSRD}(n=242) & \text { UAS }(n=101) & \text { Total }(n=466) \\ \text { Promedio de medicamentos disponibles }(n=36) & 19.0 & 17.8 & 17.4 & 18.0 & 17.7\end{array}$

$\mathrm{N}$ úmero de medicamentos disponibles

\begin{tabular}{rrrrrr}
$1-10$ & $5.0 \%$ & $2.4 \%$ & $3.3 \%$ & $3.0 \%$ & $3.2 \%$ \\
\hline $10-19$ & $50.0 \%$ & $62.7 \%$ & $62.0 \%$ & $53.5 \%$ & $59.2 \%$ \\
\hline $20-29$ & $45.0 \%$ & $34.9 \%$ & $34.7 \%$ & $43.5 \%$ & $37.6 \%$
\end{tabular}

$\chi^{2}=4.3 ; p=0.64$

CSU: centro de salud urbano

CSRC: centro de salud rural concentrado

CSRD : centro de salud rural disperso

UAS: unidad auxiliar de salud

tarios y antidiarreicos, antipalúdicos, hipotensores, hipoglucemiantes, antituberculosos, antipiréticos, métodos de planificación familiar y vacunas. Para cada grupo se calcularon las medias y las proporciones de medicamentos disponibles. Las diferencias de proporciones se evaluaron con el estadístico ji cuadrada. Finalmente, se relacionó la disponibilidad de un antibiótico inyectable (penicilina) con la existencia del material necesario para su aplicación (jeringas, algodón, alcohol) y con la intención de demostrar que la disponibilidad del medicamento puede no ser suficiente para cerrar el círculo del abastecimiento de medicamentos en las unidades de primer nivel. Todo el proceso de análisis se realizó con el paquete estadístico Stata, versión 5.0.

\section{Resultados}

Las unidades visitadas en este estudio contaban, en promedio, en el momento de la inspección, con 18 de los 36 medicamentos incluidos en la lista, con diferencias no significativas entre los distintos tipos de unidad (cuadro I). Llama la atención, sin embargo, que $5 \%$ de los centros de salud urbanos contaran tan sólo con 10 o menos de los medicamentos de la lista y que ningún centro contara con los 36 medicamentos.

Como se muestra en el cuadro II, entre las tres regiones se detectaron diferencias significativas en el número promedio de medicamentos disponibles al momento de la visita. Los estados $\mathrm{PAC}_{3}$ fueron los que presentaron un número promedio de medicamentos más elevado (18.5).

Al desagregar la información por medicamentos específicos (cuadro III), se detectaron desabastos importantes de ciertos antibióticos, de los antifímicos y de los antipalúdicos. Destaca, por ejemplo, el hecho de que más de $40 \%$ de las unidades no contara con ampicilina y que esa misma proporción de unidades no contara con penicilina. Cabe señalar, además, que estos medicamentos por lo general no pueden sustituirse. Sin embargo, también es importante mencionar que las unidades abastecidas contaban con existencias aceptables.

A un abastecimiento deficiente de antibióticos se agregan deficiencias en algunos materiales necesarios para su administración, lo que se traduce en lo que hemos dado en llamar "desabastecimiento funcional". Tal es el caso de las penicilinas inyectables, que requieren de jeringas, algodón y alcohol (cuadro IV).

Todavía más alarmantes son las cifras de antifímicos. Al momento de la visita de inspección, por

\section{Cuadro II \\ Promedio de medicamentos ESENCIALES DISPONIBLES SEGÚN REGIÓN. Secretaría de Salud, México, 1996-1997}

\begin{tabular}{lccc} 
Región & Media & Desviación estándar & $\mathrm{N}$ \\
$\mathrm{PAC}_{1}$ & 16.7 & 4.9 & 71 \\
\hline $\mathrm{PAC}_{2}$ & 17.2 & 4.1 & 164 \\
\hline $\mathrm{PAC}_{3}$ & 18.5 & 4.4 & 210
\end{tabular}

0 neway $p<0.02$

$\mathrm{PAC}_{1}$ : Programa de Ampliación de Cobertura de los estados de Chiapas, Guerrero, $\mathrm{H}$ idalgo, $\mathrm{O}$ axaca

PAC : Programa de A mpliación de C obertura de los estados de $C$ ampeche, Michoacán, N ayarit, Puebla,Veracruz, Yucatán, Zacatecas

$\mathrm{PAC}_{3}$ : Programa de A mpliación de Cobertura de los Estados de Chihuahua, Durango, Guanajuato, México, Q uerétaro, San Luis Potosí, Sinaloa 


\section{Cuadro III \\ Disponibilidad de Medicamentos esenciales en UNidAdes de PRIMER Nivel de ATENCIÓN. Secretaría de Salud, México, 1996-1997}

\begin{tabular}{|c|c|c|c|c|}
\hline M edicamentos & $\begin{array}{l}\text { Unidades sin } \\
\qquad \text { No. }\end{array}$ & $\begin{array}{c}\text { Medicamentos } \\
\%\end{array}$ & $\begin{array}{l}\text { No. de medicamentos en } \\
\text { mediana }\end{array}$ & $\begin{array}{c}\text { Unidades con existencias } \\
\text { IC } 95 \%\end{array}$ \\
\hline \multicolumn{5}{|l|}{ Antibióticos-Sulfas } \\
\hline A mpicilina susp. $60 \mathrm{ml}$ & 198 & 42.7 & 29 & $24-35$ \\
\hline Ampicilina tab. $250 \mathrm{mg}$ & 268 & 57.9 & 35 & $30-45$ \\
\hline Eritromicina susp. $250 \mathrm{mg}$ & 222 & 48.2 & 20 & $18-21$ \\
\hline Penicilina GP 800,000 U & 236 & 51.0 & 80 & $50-100$ \\
\hline Penicilina G P 1,200,000 U & 210 & 45.5 & 30 & $22-35$ \\
\hline Penicilina G P 400,000 U & 191 & 41.3 & 100 & $90-118$ \\
\hline Trimetoprim/sulfametoxazol susp. & 147 & 31.6 & 30 & $25-35$ \\
\hline \multicolumn{5}{|l|}{ Analgésicos-antipiréticos } \\
\hline Ácetaminofén susp. $15 \mathrm{ml}$ & 159 & 34.3 & 37 & $30-46$ \\
\hline \multicolumn{5}{|l|}{ Antiparasitarios-antidiarreicos } \\
\hline Metronidazol susp. $120 \mathrm{ml}$ & 144 & 31.0 & 28 & $23-31$ \\
\hline Albendazol tab. $200 \mathrm{mg}$ & 189 & 41.0 & 40 & $30-53$ \\
\hline Suero oral sobres & 51 & 11.0 & 250 & $200-250$ \\
\hline \multicolumn{5}{|l|}{ Antifímicos } \\
\hline Estreptomicina & 447 & 97.4 & 8 & $3-23$ \\
\hline Acido isonicotínico tab. $100 \mathrm{mg}$ & 437 & 95.2 & 12 & $5-121$ \\
\hline Etambutol tab. $400 \mathrm{mg}$ & 412 & 89.6 & 7 & $3-10$ \\
\hline Rifampicina cáps. 300 mg & 433 & 94.3 & 25 & $3-56$ \\
\hline Rifampicina susp. 120 ml & 453 & 98.7 & 1 & $1-2$ \\
\hline Pirazinamida tab. $500 \mathrm{mg}$ & 426 & 92.8 & 5 & $4-19$ \\
\hline \multicolumn{5}{|l|}{ Hipoglucemiantes } \\
\hline Tolbutamida tab. $500 \mathrm{mg}$ & 110 & 23.8 & 23 & $20-30$ \\
\hline Glibenclamida tab. 5 mg & 95 & 20.5 & 27 & $24-30$ \\
\hline Clorpropamida tab. $250 \mathrm{mg}$ & 248 & 53.7 & 14 & $10-19$ \\
\hline \multicolumn{5}{|l|}{ Antihipertensivos } \\
\hline Propranolol tab. 40 mg & 116 & 24.9 & 27 & $22-30$ \\
\hline Clorotiazida tab. $250 \mathrm{mg}$ & 215 & 46.4 & 18 & $15-20$ \\
\hline Captopril tab. $25 \mathrm{mg}$ & 375 & 81.0 & 8 & $5-10$ \\
\hline Furosemida tab. $40 \mathrm{mg}$ & 253 & 54.5 & 11 & $8-14$ \\
\hline \multicolumn{5}{|l|}{ Antipalúdicos } \\
\hline Cloroquina tab. $150 \mathrm{mg}$ & 287 & 62.8 & 45 & $30-60$ \\
\hline Primaquina tab. $5 \mathrm{mg}$ & 381 & 83.4 & 24 & $11-30$ \\
\hline Primaquina tab. $15 \mathrm{mg}$ & 400 & 87.7 & 20 & $10-26$ \\
\hline \multicolumn{5}{|l|}{ Planificación familiar } \\
\hline N oretisterona y etinilestradiol tab. & 93 & 20.0 & 30 & $26-35$ \\
\hline Preser vativos caja 100 & 112 & 24.1 & 3 & $2-4$ \\
\hline Dispositivo intrauterino (DIU) & 66 & 14.2 & 5 & $4-6$ \\
\hline \multicolumn{5}{|l|}{ Vacunas } \\
\hline$B C G$ & 138 & 29.7 & 4 & $3-5$ \\
\hline Antipoliomielítica & 108 & 23.3 & 4 & $4-6$ \\
\hline Antisarampionosa & 144 & 31.0 & 4 & $3-5$ \\
\hline DPT & 128 & 27.6 & 4 & $3-5$ \\
\hline Toxoide tetánico & 113 & 24.4 & 5 & $4-6$ \\
\hline Antirrábica humana & 369 & 80.2 & 5 & $3-7$ \\
\hline \multicolumn{5}{|l|}{ Material de Curación } \\
\hline Jabón neutro & 317 & 68.5 & 5 & 3-7 \\
\hline Jeringas desechables & 54 & 11.6 & 120 & $100-150$ \\
\hline Equipo venoclisis & 68 & 14.7 & 20 & $15-20$ \\
\hline Guantes quirúrgicos & 79 & 17.0 & 25 & $20-30$ \\
\hline Algo dón paquete $300 \mathrm{~g}$ & 68 & 14.6 & 3 & $3-4$ \\
\hline Alcohol 96 grados & 132 & 28.5 & 5 & $5-10$ \\
\hline Gasa estéril paquete & 208 & 44.8 & 4 & $3-5$ \\
\hline Cloruro de benzalconio & 153 & 33.0 & 4 & $3-4$ \\
\hline Sutura de seda varios calibres & 135 & 29.1 & 11 & $10-13$ \\
\hline Tela adhesiva & 89 & 19.1 & 4 & $4-5$ \\
\hline
\end{tabular}




\section{Cuadro IV \\ Porcentaje de Unidades de PRIMER NIVEL DE ATENCIÓN DE LA SSA CON PENICILINA INYECTABLE Y SU RELACIÓN CON EL MATERIAL NECESARIO PARA SU ADMINISTRACIÓN. Secretaría de Salud, México, 1996-1997}

\begin{tabular}{cc} 
& \multicolumn{2}{c}{ Penicilina inyectable } \\
\cline { 3 - 3 } & $\mathrm{Si} \quad \mathrm{No}$
\end{tabular}

Jeringas desechables

\begin{tabular}{lll} 
Sí & 90.0 & 85.9 \\
\hline No & 10.0 & 14.1
\end{tabular}

Algodón

\begin{tabular}{lll} 
Sí & 89.3 & 80.3 \\
\hline No & 10.7 & 19.7
\end{tabular}

Alcohol

\begin{tabular}{lll} 
Sí & 78.3 & 62.7 \\
\hline No & 21.7 & 37.3
\end{tabular}

Combinación de insumos*

\begin{tabular}{ccc} 
Uno & 8.0 & 13.4 \\
\hline Dos & 26.8 & 37.4 \\
\hline Tres & 65.2 & 49.2
\end{tabular}

* Se refiere a la combinación de jeringas, algodón y alcohol

ejemplo, 97\% de las unidades no contaban con un solo ejemplar de estreptomicina, 95\% no tenía disponible ácido isonicotínico y $92 \%$ no contaban con pirazinamida.

Casi todos los estados beneficiarios del PAC presentaron en 1996 casos de paludismo, con mayor o menor frecuencia. ${ }^{7}$ Sin embargo, el abasto de antipalúdicos fue muy bajo. Más de $80 \%$ de las unidades no contaban con tabletas de $5 \mathrm{mg}$ de primaquina y más de $60 \%$ no contaban con cloroquina.

Otra cifra que llama la atención es la del acetaminofén, un analgésico-antipirético particularmente útil en la atención de los niños menores de cinco años. De acuerdo con los datos de este estudio, en 34\% de las unidades no se contaba con este medicamento al momento de la inspección.

Las sales de rehidratación oral se encontraron en 90\% de los centros de salud, y los antiparasitarios (albendazol y metronidazol), en alrededor de $60 \%$ de las unidades.

Después de las sales de rehidratación oral, los métodos anticonceptivos fueron los productos más frecuentemente encontrados. La disponibilidad de pro- ductos hormonales fue de $80 \%$; la de dispositivos intrauterinos, de $85 \%$, y la de preservativos, de $76 \%$.

El abasto de biológicos varió considerablemente, dependiendo del tipo de vacuna. Así, la vacuna antipoliomielítica se encontró disponible en $77 \%$ de las unidades, mientras que la vacuna antisarampionosa sólo estuvo disponible en $69 \%$ de las unidades.

El desabasto de hipotensores podría calificarse de importante, ya que 46,81 y $54 \%$ de las unidades no contaban con clorotiazida, captopril y furosemide, respectivamente.

El abastecimiento de hipoglicemiantes orales fue aceptable, ya que se encontraron disponibles en más de $75 \%$ de las unidades, con excepción de la clorpropamida, que sólo se encontró en $46 \%$ de los centros de salud.

Finalmente, en las visitas a las unidades se registró también la disponibilidad de 10 materiales de curación: jabón neutro, jeringas desechables, equipo de venoclisis, guantes quirúrgicos, algodón, alcohol, gasa estéril, cloruro de benzalconio, hilos para sutura y tela adhesiva. Su abastecimiento fue relativamente aceptable, excepto para el jabón neutro, las gasas, el cloruro de benzalconio y los hilos para sutura, que estaban ausentes en $68,45,33$ y 29\% de las unidades, respectivamente. Estas cifras ilustran muy claramente los problemas a los que se enfrentan las unidades de primer nivel para atender las lesiones, que hoy constituyen, junto con los homicidios, la principal causa de pérdida de años de vida saludables en todo el país. ${ }^{8}$

$\mathrm{Al}$ desagregar la información por grupos terapéuticos -antibióticos, vacunas, antipalúdicos, hipoglucemiantes orales, hipotensores, material de curación y métodos de planificación familiar- y por tipo de unidad (cuadro V), se detectó un buen abastecimiento general de métodos de planificación familiar e hipoglucemiantes orales, sin diferencias significativas entre los distintos tipos de unidad. En donde se detectaron problemas serios de abastecimiento fue sobre todo en los grupos de antibióticos, antipalúdicos e hipotensores, con diferencias significativas entre los distintos tipos de unidad en los primeros dos casos. El promedio de antibióticos disponibles para todas las unidades fue de 3.8. de un máximo posible de siete. Las diferencias por tipo de unidad, sin embargo, fueron importantes. Los CSRC, por ejemplo, contaban, en $48 \%$ de los casos, con dos o menos antibióticos disponibles al momento de la visita. En contraste, las UAS contaban, en 55\% de los casos, con entre cinco y siete antibióticos disponibles.

El promedio de antipalúdicos disponibles para todos los tipos de unidad fue de 0.64 , de un máximo posible de tres, con diferencias importantes por tipo 


\section{Cuadro V}

Disponibilidad de medicamentos eSEnCIALES POR UNidADES DE PRIMER NIVEL De ATENCIÓN. Secretaría de Salud, México, 1996-1997

\begin{tabular}{|c|c|c|c|c|c|}
\hline M edicamentos/unidades & $\operatorname{CSU}(n=40)$ & $\operatorname{CSRC}(n=83)$ & $\operatorname{CSRD}(n=242)$ & UAS $(n=101)$ & Total $(n=466)$ \\
\hline Promedio antibióticos ( $n=7$ ) & 3.5 & 3.0 & 3.8 & 4.4 & 3.8 \\
\hline $\mathrm{N}$ inguno & $12.5 \%$ & $20.5 \%$ & $8.2 \%$ & $5.0 \%$ & $10.1 \%$ \\
\hline $1-2$ & 20.0 & 27.7 & 19.0 & 13.8 & 19.5 \\
\hline $3-4$ & 32.5 & 19.3 & 33.5 & 25.7 & 29.2 \\
\hline $5-7$ & 35.0 & 32.5 & 39.3 & 55.5 & 41.2 \\
\hline \multicolumn{6}{|l|}{$\chi^{2}=29.1 ; p=0.001$} \\
\hline Promedio vacunas $(n=6)$ & 4.6 & 4.4 & 3.7 & 3.3 & 3.8 \\
\hline N inguna & $7.5 \%$ & $12.0 \%$ & $18.6 \%$ & $26.7 \%$ & $18.2 \%$ \\
\hline $1-2$ & 2.5 & 1.2 & 6.6 & 5.0 & 5.0 \\
\hline 3-4 & 15.0 & 18.1 & 21.5 & 19.8 & 20.0 \\
\hline $5-6$ & 75.0 & 68.7 & 53.3 & 48.5 & 56.8 \\
\hline \multicolumn{6}{|l|}{$\chi^{2}=19.5 ; p<0.021$} \\
\hline Promedio antipalúdicos $(n=3)$ & 0.47 & 0.97 & 0.50 & 0.78 & 0.64 \\
\hline N inguno & $70.0 \%$ & $49.4 \%$ & $67.4 \%$ & $53.5 \%$ & $61.4 \%$ \\
\hline 1 & 17.5 & 24.1 & 20.6 & 26.7 & 22.3 \\
\hline 2 & 7.5 & 6.0 & 5.8 & 7.9 & 6.4 \\
\hline 3 & 5.0 & 20.5 & 6.2 & 11.8 & 9.8 \\
\hline \multicolumn{6}{|l|}{$\chi^{2}=21.3 ; p<0.011$} \\
\hline Promedio hipoglucemiantes $(n=3)$ & 2.1 & 2.1 & 2.0 & 2.0 & 2.0 \\
\hline $\mathrm{N}$ inguno & $2.5 \%$ & $2.4 \%$ & $6.6 \%$ & $5.0 \%$ & $5.2 \%$ \\
\hline 1 & 22.5 & 22.9 & 19.0 & 22.7 & 20.8 \\
\hline 2 & 40.0 & 39.7 & 43.0 & 43.6 & 42.3 \\
\hline 3 & 35.0 & 35.0 & 31.4 & 28.7 & 31.7 \\
\hline \multicolumn{6}{|l|}{$\chi^{2}=4.5 ; p<0.8$} \\
\hline Promedio hipotensores $(n=4)$ & 2.0 & 2.0 & 2.0 & 1.7 & 1.9 \\
\hline N inguno & $7.5 \%$ & $8.4 \%$ & $7.8 \%$ & $11.8 \%$ & $8.8 \%$ \\
\hline $1-2$ & 57.5 & 57.8 & 59.1 & 66.4 & 60.3 \\
\hline $3-4$ & 35.0 & 33.8 & 33.1 & 21.8 & 30.9 \\
\hline \multicolumn{6}{|l|}{$\chi^{2}=11.2 ; p<0.5$} \\
\hline Promedio material curación $(n=10)$ & 7.4 & 6.9 & 7.3 & 6.9 & 7.1 \\
\hline $0-5$ & $17.5 \%$ & $24.1 \%$ & $16.5 \%$ & $16.8 \%$ & $18.0 \%$ \\
\hline $6-10$ & 82.5 & 75.9 & 83.5 & 83.2 & 82.0 \\
\hline \multicolumn{6}{|l|}{$\chi^{2}=2.5 ; p<0.5$} \\
\hline \multicolumn{2}{|c|}{ Promedio métodos planificación familiar $(n=3) 2.5$} & 2.3 & 2.4 & 2.5 & 2.4 \\
\hline N inguno & $5.0 \%$ & $7.2 \%$ & $2.9 \%$ & $4.0 \%$ & $4.1 \%$ \\
\hline 1 & 5.0 & 6.1 & 12.8 & 6.0 & 9.4 \\
\hline 2 & 22.5 & 32.5 & 29.4 & 22.7 & 27.9 \\
\hline 3 & 67.5 & 54.2 & 54.9 & 67.3 & 58.6 \\
\hline
\end{tabular}

CSU: centro de salud urbano

CSRC: centro de salud rural concentrado

CSRD : centro de salud rural disperso

UAS: unidad auxiliar de salud 
de unidad. El mejor abastecimiento se presentó en los CSRC, mientras que los CSU presentaron las cifras más pobres: $70 \%$ de estos centros no contaban con ningún antipalúdico al momento de la visita de inspección.

\section{Discusión}

El abastecimiento adecuado de medicamentos es un factor crucial en la cadena de la atención de la salud. De hecho, en muchos países en desarrollo la calidad de la atención es sinónimo de disponibilidad de medicamentos. Sin embargo, los problemas a este respecto siguen siendo enormes. Los recursos que se dedican a la compra de medicamentos esenciales en estos países suelen ser insuficientes y tienden a concentrarse en las unidades de segundo y tercer nivel de atención. A esta insuficiencia de recursos deben agregarse los problemas de planeación, adquisición, transporte, almacenamiento y distribución de estos insumos.

En México, además de la insuficiencia de recursos para atender las necesidades de salud de la población no asegurada, se sabe de la existencia de desabastecimientos de medicamentos y otros insumos que tienen su origen en problemas de planeación, licitación y compra, almacenamiento y distribución, como se demostró en un estudio no publicado, realizado por la Dirección General de Evaluación y Seguimiento de la SSA en 1996. ${ }^{*}$

Por lo que se refiere a la planeación, el cálculo de los requerimientos estatales y jurisdiccionales de medicamentos y material de curación, sigue haciéndose sobre la base de consumos históricos y no refleja la demanda real, ya que no toma en cuenta ni las variaciones demográficas ni los cambios epidemiológicos.

Por lo que toca a la licitación, compra de medicamentos y material de curación, el tiempo real de duración de las licitaciones tiende a ser excesivo -en ocasiones hasta de seis meses-; las medidas implantadas para evitar los incumplimientos de los proveedores (fianzas) no suelen ponerse en práctica, y con frecuencia la federación asigna a los estados cantidades que no se ajustan a lo solicitado y no radica a tiempo el presupuesto para el pago a los proveedores de medicamentos y material de curación.

En relación con el almacenamiento y la distribución, cabe mencionar que las áreas centrales de la

\footnotetext{
* Dirección General de Evaluación y Seguimiento, Secretaría de Salud. Evaluación del Programa de Apoyo a Servicios de Salud para Población Abierta (PASSPA). México, D.F.: SSA, 1996. Documento no publicado.
}

SSA no suelen proporcionar información oportuna a los estados sobre los insumos asignados; los almacenes tanto jurisdiccionales como hospitalarios son inadecuados en términos de espacio, control de temperatura ambiental y acceso a insumos de almacenamiento (racks, refrigeradores); el control y la rotación de inventarios es ineficiente, lo cual provoca fugas y desperdicios -en la mayor parte de las jurisdicciones se reportan desechos regulares anuales de alrededor de $10 \%$ de lo abastecido-, y las entregas de los medicamentos y material de curación tienden a ser insuficientes y tardías.

Los resultados sobre el abastecimiento de medicamentos esenciales en las unidades de primer nivel de atención de la SSA, que aquí se presentan, son un fiel reflejo de la persistencia de estos problemas. $\mathrm{Al}$ momento de las visitas de inspección de las unidades que se realizaron en este estudio, se detectó un problema de abastecimiento general que podría calificarse de serio. Los problemas de abastecimiento de antibióticos, antifímicos y antipalúdicos fueron particularmente graves. No obstante, el desabastecimiento de los antifímicos podría explicarse en parte por la obligación de solicitar dichos medicamentos a los niveles centrales estatales hasta después del diagnóstico de cada caso.

El abastecimiento de sales de rehidratación oral, métodos de planificación familiar y biológicos fue relativamente aceptable. Sin embargo, no todos los biológicos se encontraron en las cantidades deseadas, aunque se sabe que las Semanas Nacionales de Salud garantizan una distribución y aplicación adecuadas de estos medicamentos -lo mismo que de los antiparasitarios-, lo que ha permitido alcanzar cifras de cobertura excelentes, sobre todo en menores de cinco años de edad.

Finalmente, el abastecimiento de medicamentos para el tratamiento de la hipertensión arterial y la diabetes mellitus presentó valores intermedios, mientras que el abastecimiento de material de curación fue pobre, en particular el de jabón y gasas.

La escasez de medicamentos y material de curación fue particularmente crítica en las unidades de los estados $\mathrm{PAC}_{1}$, que en promedio presentaron 16.7 medicamentos de los 36 incluidos en la lista de referencia.

Cabe señalar que la definición que se utilizó aquí de abastecimiento -presencia de por lo menos un ejemplar de algún medicamento- es muy conservadora, $\mathrm{y}$ que en todas las unidades en donde se detectó desabastecimiento, éste significó ausencia total de alguno o varios de los medicamentos estudiados. Se trata, además, de medicamentos para padecimientos que 
contribuyen de manera muy importante a la carga de la enfermedad en el país.

No es de sorprender, por lo tanto, que una de las principales causas de no utilización de las unidades de primer nivel de atención de la SSA, reportadas en la ENSA II, sea la carencia de medicamentos y material de curación, sobre todo si se considera que es la población con menor capacidad de pago la que se atiende en este tipo de unidades. Una proporción importante de esta población, de hecho, difícilmente podría surtir su receta en las farmacias privadas de su pueblo, aun en el caso de que su poblado contara con este tipo de servicio, debido a las barreras financieras. ${ }^{9}$ Estas mismas personas difícilmente regresarían a hacer uso de unidades de salud que no tienen la capacidad resolutiva que les exige el nivel de ingresos de su población de responsabilidad.

La SSA, en general, y el Programa de Ampliación de Cobertura, en particular, deberán hacer un esfuerzo enorme por acabar con los cuellos de botella que impiden un abastecimiento adecuado de medicamentos esenciales en las unidades de primer nivel de atención. De otra manera, todos los demás esfuerzos por atender las necesidades de salud de las poblaciones más marginadas del país resultarán inútiles, ya que el acceso a los medicamentos constituye la pieza final indispensable de la enorme cadena de la atención a la salud.

\section{Referencias}

1. Reich MR. Essential drugs: Economics and po litics in international health. Health Pol 1987;8:39-57.

2. Facha J. Cuadros básicos de insumos del sector salud en México. En: Memorias de la 1a. Conferencia Latinoamericana sobre Políticas Farmacéuticas y Medicamentos Esenciales. México, D.F.: O MS/O PS/SSA/IN SP, 1981:10-14.

3.C uadro Básico de Medicamentos del Sector Público. Diario 0 ficial 1977, 2 de diciembre:1-3.

4. Kravzov J,Altagracia M. C hanges in the Mexican essential drug list.J Soc Admn Pharmacy 1994;11:150-153.

5. Secretaría de Salud. A cuerdo por el que se instituye el Cuadro Básico de Insumos del Sector Salud. Diario 0 ficial 1983, 9 de junio:22-24.

6. Secretaría de Salud. Encuesta N acional de Salud II. México, D.F.: Secretaría de Salud, 1994.

7. Epidemiología. Sistema Nacional de Vigilancia Epidemiológica 1997;14 (semana 53).

8. Lozano R. El peso de la enfermedad en México: avances y desafíos. En: Frenk J, ed. 0 bservatorio de la salud: necesidades, servicios y políticas. México, D.F.: Fundación Mexicana para la Salud,1992:23-61.

9. Leyva-Flores R, Erviti-Erice J, Kageyama-Escobar ML,Arredondo A. Prescripción, acceso y gasto en medicamentos entre usuarios de servicios de salud en México. Salud Publica Mex 1998;40(1):24-31. 